\title{
Restarting gastrointestinal endoscopy in the deceleration and early recovery phases of COVID-19 pandemic: Guidance from the British Society of Gastroenterology
}

\author{
Authors: Colin J Rees, ${ }^{\mathrm{A}}$ James E East, ${ }^{\mathrm{B}}$ Kofi Oppong, ${ }^{\mathrm{C}}$ Andrew Veitch, ${ }^{\mathrm{D}}$ Mark McAlindon, ${ }^{\mathrm{E}}$ John Anderson, ${ }^{\mathrm{F}}$ \\ Bu Hayee, ${ }^{G}$ Cathryn Edwards, ${ }^{H}$ Alastair McKinlay ${ }^{\mathrm{I}}$ and Ian Penman ${ }^{\mathrm{C}}$
}

\begin{abstract}
Many non-emergency clinical services were suspended during COVID-19 pandemic peak. It is essential to develop a plan for restarting services following the peak. It is equally important to protect patients and staff and to use resources and personal protective equipment (PPE) efficiently. The British Society of Gastroenterology Endoscopy Committee and Quality Improvement Programme has produced guidance on how a restart can be safely delivered. Key recommendations include the following: all patients should have need for endoscopy assessed by senior clinicians and prioritised according to criteria we have outlined; once the need for endoscopy is confirmed, patients should undergo telephone screening for symptoms using systematic questionnaires; all outpatients should undergo RT-PCR testing for COVID-19 virus 1-3 days prior to endoscopy; and PPE should be determined by patient risk stratification, the nature of the procedure and the results of testing. While this guidance is tailored to endoscopy services, it could be adapted for any interventional medical discipline.
\end{abstract}

KEYWORDS: Covid-19, pandemic, endoscopy, British Society of Gastroenterology

DOI: 10.7861/clinmed.2020-0296

Authors: Aprofessor of gastroenterology, Newcastle University, Newcastle Upon Tyne, UK and consultant gastroenterologist, South Tyneside and Sunderland NHS Foundation Trust, South Shields, UK: ${ }^{B}$ Consultant gastroenterologist, Oxford University Hospitals NHS Foundation Trust, Oxford, UK; ' Consultant gastroenterologist, Newcastle upon Tyne Hospitals NHS Foundation Trust; ${ }^{\mathrm{D}}$ consultant gastroenterologist, The Royal Wolverhampton NHS Trust,

Wolverhampton, UK; ${ }^{\mathrm{E}}$ Consultant gastroenterologist, Sheffield Teaching Hospitals NHS Trust, UK; ${ }^{F}$ consultant gastroenterologist, Gloucestershire Hospitals NHS Foundation Trust, UK; ${ }^{G}$ Consultant gastroenterologist, King's College Hospital NHS Foundation Trust, London, UK; Hpresident, British Society of Gastroenterology (BSG) and consultant gastroenterologist, Torbay and South Devon NHS Foundation Trust, Torbay, UK; Ipresident-elect, BSG and consultant gastroenterologist, Aberdeen Royal Infirmary, Aberdeen, UK; ' vice president endoscopy, BSG and consultant gastroenterologist, Royal Infirmary of Edinburgh, UK

\section{Background}

The COVID-19 pandemic has had greater impact on global healthcare than almost any single event in living memory. The impact upon health services has been unparalleled, with the huge pressure of COVID-19 workload leading to suspension and reorganisation of services. Elective and urgent services, including gastrointestinal (GI) endoscopy, were largely suspended during the peak phase of the pandemic in order to allow redeployment of resources and to protect patients and the workforce. Protection of patients and staff from COVID-19 is of particular relevance to GI endoscopy, as many endoscopic procedures are aerosolgenerating. ${ }^{1-3}$

The British Society of Gastroenterology (BSG) issued guidance ${ }^{4}$ regarding emergency and essential procedures during the peak phase of the pandemic. Other endoscopy organisations published similar guidance. ${ }^{5}$ The short-term delays to services outlined in these guidance documents would represent a considerable risk to patient safety if prolonged. These risks are particularly important for a diagnostic speciality such as GI endoscopy which has cancer diagnosis as one of its main functions. Data from the UK National Endoscopy Database (NED), ${ }^{6}$ a data warehousing programme led by the Royal College of Physicians, indicate that total endoscopic activity fell rapidly to $5 \%$ of normal levels during the peak phase of the COVID-19 epidemic in the UK, from approximately 35,000 per week to 1,700 for the week ending 13 April. ${ }^{6}$ Modelling studies suggest that delays to cancer diagnoses and treatment could potentially be responsible for up to 7,000 additional deaths in England and over 30,000 in the United States. ' Having passed the initial peak and entered the deceleration and early recovery phases of the pandemic, ${ }^{8,9}$ it is therefore important that strategies for safe restoration of clinical services, including diagnostics, are developed.

The following guidance was developed by the BSG Endoscopy committee, led by the BSG Endoscopy Quality Improvement Programme. It has been issued to guide the safest possible restart of GI endoscopy services at scale. This guidance was downloaded over 2,000 times within 24 hours of becoming available on the BSG website (Fig 1), indicating the appetite for such guidance from clinical teams. While this guidance relates to GI endoscopy, similar approaches could be applied to any interventional medical speciality. 


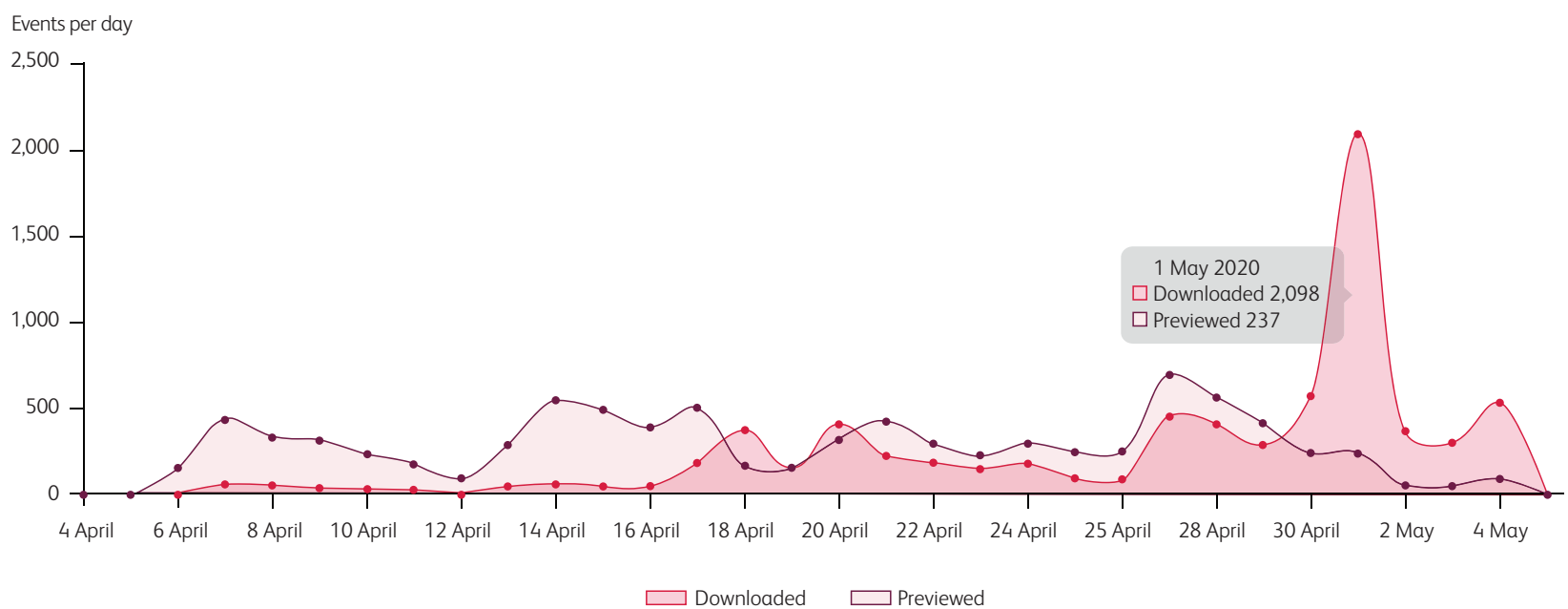

Fig 1. Downloads of all British Society of Gastroenterology (BSG) documents (endoscopy and non-endoscopy) from BSG website between 4 April 2020 and 4 May 2020.

\section{General principles}

The BSG has already published strategies for endoscopy services in the build-up or acceleration phase of the current epidemic, ${ }^{10}$ and a suite of service recovery documents giving a pragmatic 'toolkit' for GI unit operations, including endoscopy, during COVID-19. ${ }^{4}$

Following the cessation of all non-emergency and essential endoscopy activity in March to April 2020, a plan for how endoscopy services might safely restart was developed. This covered the deceleration and early recovery phases of the epidemic and was designed to be read alongside the BSG Service Recovery framework documents. ${ }^{4}$ Different areas of the UK are likely to move through the phases of the COVID-19 epidemic at different rates of progression and at different time points. The capability and capacity to restore endoscopy practice will vary across the UK and the guidance was designed to be adaptable to match up with local capacity to deliver. It should also be noted that the COVID-19 landscape is rapidly changing, and the guidance was not designed to be exhaustive but to provide practical advice for endoscopy teams looking to restart services. The guidance is likely to be adapted over time and in response to feedback, incorporating greater detail in some areas of practice. It is anticipated that further guidance will be published for future stages of the pandemic. Guidance was developed based upon review of the best available evidence and expert consensus opinion.

In resuming endoscopy services, the following principles were applied.

$>$ Optimising patient outcomes.

$>$ Protecting patients.

$>$ Protecting staff.

> Correctly and efficiently utilising resources so that the maximum benefit is delivered for the greatest number of patients.

> Addressing those patients who had endoscopy appointments 'suspended' before the COVID-19 peak (many of whom may have known that they were at significant risk of a cancer diagnosis or other serious diagnosis).

> Ensuring that all referrals are triaged by senior decision-makers and balance clinical need and potential benefit.
> Continuing mitigation strategies that are clinically driven by the reprioritisation of deferred referrals as capacity allows.

Above all, the guidance followed the principle that decisions should be guided by a strong ethical framework such as that set out in the Royal College of Physicians guidance Ethical dimensions of COVID-19 for frontline staff, which embodies the fundamental principle that all decisions should be accountable, inclusive, transparent, reasonable and responsive. ${ }^{11}$

\section{Protecting patients and staff}

In order for endoscopy services to return at significant volume, it is essential that both the public and clinical staff are confident that they are as safe as possible and that the risk of COVID-19 infection has been managed and reduced as much as is feasible (within the constraints of current knowledge). The public have correctly avoided unnecessary contact with health services during the build-up and peak phases of the epidemic and, therefore, need to be reassured that attending for endoscopy does not compromise their safety or put health services under undue pressure. The safety of staff is of paramount importance. The infection poses a potential risk to their health and also carries risk of death, so the correct degree of protection is essential.

Resumption of endoscopy activity must include strict infection control measures to reduce spread of the infection, as described in recent publications from endoscopy centres in China and Italy, ${ }^{12-15}$ protection of patients and staff by appropriate testing for COVID-19, and meticulous contact tracing. The availability of a guaranteed supply of enough appropriate personal protective equipment (PPE) is also crucial.

\section{COVID-19 screening testing to facilitate the resumption of endoscopy services}

Many endoscopy procedures are aerosol-generating and, when undertaken on individuals who are infected with COVID-19, result in a significant risk of infection to other patients and endoscopy staff. The requirement for Level 2 (enhanced) PPE, 
however, reduces the capacity for endoscopy procedures by up to $50 \%{ }^{16-18}$ and places a high demand on supplies of PPE, especially FFP3 respirators and fluid-resistant gowns, which may stretch available resources. While the availability of a guaranteed supply of appropriate PPE is, therefore, an essential requirement for the resumption of activity, strategies to improve productivity and safety while making most effective use of PPE also need to be considered. An additional issue is the time required for more comprehensive room cleaning and air circulation in endoscopy rooms in between procedures, which significantly slows endoscopy activity.

One solution to this is to develop a comprehensive COVID-19 screening strategy for endoscopy procedures. This will allow lower levels of PPE to be worn for lower-risk procedures and increase room throughput for outpatient endoscopy, while conserving PPE and maintaining patient and staff safety. No accepted protocol for rapid, accurate pre-endoscopy testing currently exists. A number of possible strategies have been suggested, but all have potential problems. Screening for symptoms and the use of testing for infection likely offer the best routes to allow resumption of endoscopy activity. We propose a pre-endoscopy screening strategy as developed by Hayee et al (not yet published) which screens patients on the basis of history and clinical features along with RT-PCR-based testing for COVID-19 virus pre-procedure. Where patients are COVID-positive or deemed high risk, then all procedures, other than emergencies, should be deferred until the patient has recovered from the viral illness.

It is also important that due consideration is given to the optimal environment in which to deliver endoscopy services. Where possible, for example where more than one unit exists within an organisation, elective services should be delivered at cold or 'COVID-minimised' sites; if that is not feasible, then separation of COVID and non-COVID patients is essential. Units could have 'COVID-minimised' and 'hot' days of the week, or could prioritise inpatients and COVID-positive patients in separate rooms, prioritised to the afternoon to allow deep cleaning and settling of the rooms overnight.

Linear patient flow through the unit, with no crossing of COVID-positive and COVID-negative pathways and a separate entrance and exit, is essential. Keeping known or suspected COVID patients out of COVID-minimised areas, for example scoping in a designated COVID operating theatre or at the patient's bedside, is also an option. A slower throughput of patients is necessary to reduce the risk of positive and negative patients meeting staff. Enhanced screening and testing of staff is required to maintain 'COVID-minimised' units, for example checking for symptoms such as fever before work, and avoiding the rotation of staff between 'hot' and 'COVID-minimised' parts of a hospital or site. Thus, the principle of minimising risk of spreading infection by separation of COVID-negative and positive/suspected patients in time and space can be achieved in a number of ways, according to locally determined factors.

\section{PPE and infection control measures}

Appropriate PPE should be available for each type of endoscopic procedure for all staff involved and procedures should be deferred until appropriate PPE is available. The resumption of endoscopic services is critically dependent on the supply of PPE being sustainable, reliable and sufficient to meet the needs of the entire range of services involved in the care of patients with COVID-19.
Until those criteria can be demonstrably achieved it will be difficult to reinstitute endoscopy for any but the most urgent cases. Once supplies are dependable, particularly as the epidemic decelerates, then services can begin to resume. Advice from Public Health England (PHE) and the comparable agencies within the devolved administrations states that working in areas where aerosol generating procedures (AGPs) are performed requires the use of enhanced (level 2) PPE. ${ }^{19}$ This includes endoscopy units but raises the crucial question of which procedures pose the greatest risk to staff and other patients.

The overall risk to staff and patients is likely to depend on the stage of the COVID-19 infection, the viral load and the infectivity of the secretions involved. As a consequence, not all endoscopic procedures may carry the same risk to staff. The infectivity of upper airways and nasopharyngeal secretions are well established. For this reason, the requirement for enhanced (level 2) PPE for upper GI endoscopic procedures is unlikely to change in the foreseeable future. If it becomes possible to demonstrate that antibodies are protective, and this, when combined with negative viral swabs, can show that the transmission of infection is unlikely, then this might change.

The situation regarding lower GI procedures is less clear. Viral RNA can be detected in stool for several weeks, but viable virus is not present. ${ }^{20-26}$ This is consistent with viral dynamics from sputum and lung where multiple studies have shown presence of nonviable virus for prolonged periods. It is reasonable, therefore, to categorise lower GI procedures as of lower transmissibility risk than upper GI procedures. Thus, if patients have been screened and are asymptomatic for 14 days prior to endoscopy and have a negative nasopharyngeal swab, this should allow the use of less stringent infection control policies for lower GI procedures. This could facilitate higher throughput and aid service recovery and would also allow use of lower levels of PPE for lower GI procedures.

Best practice measures in infection control must also be followed, including adequate time for air exchanges in rooms and deep cleaning between procedures. This will affect capacity and appropriately spaced bookings will be necessary. Appropriate social distancing of patients and staff both pre- and postprocedure is also essential. It is important that consideration is given to other elements of endoscopy as well as the procedure itself. These might include, but not be restricted to, use of nitrous oxide:oxygen gas (Entonox), use of nasal oxygen, administration of throat spray and enemas. Further work is required to establish the risks of these interventions.

Audit data from Italy suggest that adherence to strict infection control policies, including PPE and curtailment of routine activity, is associated with low rates of transmission of infection to both patients and healthcare workers. ${ }^{15}$ Data on the effectiveness of safety measures in endoscopy are essential for quality assurances purposes: to protect the public, patients and staff; to rationalise use of PPE supplies; and to inform planning for any future outbreaks.

\section{Guidance for specific endoscopy procedures}

The importance of continuous senior decision-maker involvement in triaging and prioritisation of referrals in order to balance clinical need with available capacity and the need to monitor this balance frequently over time cannot be overstated.

The BSG issued guidance on procedures to be undertaken during the peak phase of the pandemic. These are listed in Table 1 as a reference point. 
Table 1. Emergency and essential endoscopies continued during peak phase of COVID-19

\section{Procedure}

Upper GI endoscopy

Endoscopic retrograde cholangiopancreatography (ERCP) and hepatico-pancreatico-biliary endoscopic ultrasound (EUS)

Capsule endoscopy (small bowel)

Device-assisted enteroscopy (small bowel)

Lower GI endoscopy (colonoscopy or flexible sigmoidoscopy)

\section{Indication}

Acute upper-GI bleeding (including ongoing banding of varices post-acute bleed)

Total dysphagia and food bolus obstruction

Obstructing upper-GI lesion requiring stenting or therapy

Urgent nutritional support with nasogastric/jejunal tube or percutaneous endoscopic gastrostomy (PEG)

Endoscopic vacuum therapy

All presentations of cholangitis

Obstructive jaundice, where required for significant symptoms or preoperatively

Biliary stent change if clinically indicated (asymptomatic plastic stents deferred for max 3 months, asymptomatic fully covered metallic stents deferred for max 1 year)

Post-operative complications - bile leak, stricture

Pancreatic stent for disrupted duct

Therapeutic EUS - drainage of peripancreatic collections and biliary drainage after failed ERCP

Continuous or frequent small-bowel bleeding (overt or occult) in patients who are hospitaldependent or requiring repeated hospital admissions

For therapy, for example continuous or frequent small bowel bleeding (overt or occult) in patients who are hospital-dependent or requiring repeated hospital admissions

Ongoing lower GI bleeding where interventional radiology is not possible or unsuccessful
In the deceleration and early recovery phases, endoscopy should commence with those patients who were 'deferred/paused' before the peak of the outbreak and still deemed to require endoscopic investigation, following re-triage and prioritisation by senior decision-makers. Patients whose procedures were deferred or cancelled should remain on patient tracking lists and be followed up at clinic or by telephone to review their clinical condition and possible need for endoscopy to be re-prioritised. More specific recommendations for individual GI endoscopic procedures follow.

\section{Upper GI (UGI) endoscopy}

The following groups should be considered for oesophago-gastric duodenoscopy (OGD).

Dysphagia should be verified at the point of consultant/nurse specialist triage and patients with new dysphagia should be assessed using the Edinburgh Dysphagia Score (EDS):27

$>$ EDS $\geq 3.5$ - direct to urgent OGD if appropriate and fit

$>$ EDS $<3.5$ - if $>55$ years old, plan OGD urgently as lifting of COVID restrictions allow or consider an alternative diagnostic method, eg barium studies or computed tomography (CT) if clinically appropriate.

Dyspepsia should be verified at the point of consultant/nurse specialist triage and the following criteria applied:

> Patients $>55$ years old with new dyspepsia and unexplained weight loss should proceed to OGD as urgently as lifting of COVID restrictions allow.

$>$ Patients $>55$ years old with new dyspepsia ( $<6$ months) and anaemia should proceed to OGD as urgently as lifting of COVID restrictions allow.

> Patients with an abdominal mass or $>60$ years old with abdominal pain and unexplained weight loss should have urgent CT of the thorax, abdomen and pelvis (before considering OGD).
Direct/open access OGD (for those sites that have this service) should remain suspended. Patients already referred via this pathway should be re-triaged, reviewed and managed according to their symptoms.

All surveillance of long-term conditions should be suspended, including:

> Barrett's surveillance (non-dysplastic and low-grade dysplasia)

> post-EMR surveillance (after satisfactory first OGD post-EMR)

$>$ post-radiofrequency ablation surveillance

$>$ surveillance for gastric atrophy/intestinal metaplasia

$>$ varices surveillance.

For dyspeptic patients, OGD should not be performed in the absence of alarm features - a policy of treatment with proton pump inhibitors (PPIs) and $H$ pylori testing should be undertaken as per NICE guidance. All patients with solely reflux symptoms should be given treatment with full dose PPI.

Suspected coeliac disease should be treated on the basis of serology without duodenal biopsies. For patients with a serum TTG $>10 \times$ the upper limit of normal (ULN), this has been shown to be accurate and safe, as long as there are no 'alarm' features (agreed by Prof D Sanders, chair, Health Advisory Group, Coeliac UK). Units should develop a locally agreed policy with colleagues with expertise in management of coeliac disease, especially for patients with lower levels of TTG or atypical presentations.

Follow-up endoscopy for healing of grade $C$ oesophagitis should be suspended.

\section{Endoscopic retrograde cholangiopancreatography (ERCP)}

Alternatives to ERCP should be considered for the following conditions, and it should proceed only after multidisciplinary team (MDT) discussion: 
$>$ Hilar obstruction - percutaneous biliary drainage (PTBD) can be considered in selected cases

> ampullectomy - defer unless deemed high risk of progression to malignancy over 2-3 months

$>$ difficult bile duct stones potentially requiring long procedure or cholangioscopy - consider deferring or surgery or interval stent change.

ERCP procedures should be deferred for the following conditions:

$>$ the majority of pancreatic conditions

$>$ sphincter of Oddi dysfunction

$>$ asymptomatic bile duct stones.

Hepatico-pancreatico-biliary endoscopic ultrasound (EUS)

EUS procedures should be continued for tissue acquisition in pancreaticobiliary malignancy where it will significantly influence management, although alternative (non-AGP) options for tissue acquisition can be considered prior to referral.

For the following, alternative options should be considered and EUS should only proceed after MDT discussion:

> common bile duct stones (consider magnetic retrograde cholangiopancreatography)

> assessment of neoplastic cyst - consider EUS if high risk features and recommended by specialist hepatico-pancreaticobiliary MDT

> cancer staging - only consider if recommended by specialist MDT.

EUS can be deferred for procedures including:

> dilated bile duct with normal liver function tests

$>$ non-specific abdominal pain

$>$ recurrent pancreatitis

$>$ submucosal lesions - unless there is high suspicion of malignancy and EUS is recommended by specialist MDT.

\section{Capsule endoscopy}

Capsule endoscopy can be used in the following situations, although careful assessment is required to ensure the risk of capsule retention is minimised and there is confidence in the availability of device-assisted enteroscopy or surgery in the event of capsule retention, if removal is clinically required.

> Suspected small bowel bleeding (occult and overt) in men and non-menstruating women of 60 years of age and under.

> Radiological imaging in which a possible diagnosis of a small bowel tumour is made but further supportive evidence of the diagnosis is needed.

\section{Device-assisted enteroscopy}

Device-assisted enteroscopy can be considered:

$>$ in patients with small bowel bleeding (overt or occult) requiring frequent blood and/or iron infusions

$>$ to obtain histology in patients with localised lesions (including masses and strictures) identified by capsule endoscopy or radiology.

Lower GI (LGI) endoscopy in symptomatic patients (non-bowel cancer screening)

This will include management of patients suspended during the peak with polyps where there is concern about cancer. These should be prioritised depending upon clinical risk. For patients with complex polyps, prioritisation should begin with those lesions with high grade dysplasia, rectal lesions, those with depressed components and laterally spreading tumours (LST) according to known risk features.

All LGI referrals - 2-week wait (2WW) and non-2WW - which are made to secondary care should have a quantitative faecal immunochemical test (qFIT) undertaken (allowing the level of blood in stool to be measured as this correlates with risk of malignancy) and, following review by a senior decision maker, should proceed to LGI endoscopy or CT colonography (as determined by local service availability and relevant national guidance). Updated detailed guidance from both NHSE and Scottish government is expected shortly. British Society of Gastrointestinal and Abdominal Radiology (BSGAR) advice on use of CT colonography has been published on their website. ${ }^{28}$

Where patients are referred with iron deficiency anaemia, upper GI endoscopy should be considered after lower GI investigation (at a subsequent appointment) because the former is a higher-risk AGP.

No 'straight to test' (STT) colonoscopy or flexible sigmoidoscopy procedures should be accepted without the involvement of a senior decision maker to consider risks and benefits, and the overall priority within a limited service.

The need for all disease-based surveillance (inflammatory bowel disease, post polypectomy, post cancer) should be reviewed, with all surveillance deferred to beyond the deceleration and recovery phase with subsequent gradual reintroduction in line with new British Society of Gastroenterology (BSG) guidelines (and as dictated by local capacity).

With genetic-based screening or surveillance, where the risk of delay is relatively low (eg based upon family history), defer until after deceleration and recovery phase. Where the risks of a delay in interval screening are higher, eg with Lynch syndrome, consider delaying where possible but proceed on a case-by-case basis.

Policies relating to the use of qFIT in primary care vary among the devolved nations, so relevant national policy guidance should be followed. NHS England recommend that if qFIT is $<10$ micrograms of haemoglobin per gram of faeces, do not proceed to LGI endoscopy but develop local safety net and criteria for further assessment and management based upon symptoms. The use of qFIT levels of $<10$ to inform decisions on patient investigation should be undertaken by specialists in secondary care and not solely in primary care. More detailed advice on qFIT cut off levels is expected to be published soon.

All non-cancer referrals should be considered by a senior decision maker to review the yield and value of the proposed procedure. This will be based upon evaluating the potential risks and benefits to patients of endoscopy versus symptomatic management.

With new inflammatory bowel disease (IBD), likely diagnosis should be assessed on the basis of symptoms and biomarkers, including calprotectin. Consider empirical treatment if this is low risk, but proceed to colonoscopy or flexible sigmoidoscopy where needed for diagnosis, or to inform decisions regarding the escalation of therapy.

Known IBD should be treated on the basis of symptoms or biomarkers where possible, but proceed to LGI endoscopy where clinical management will be significantly influenced, to determine progression of disease extent. 


\section{Bowel cancer screening programmes}

qFIT screening should be recommenced as determined by national policy during the deceleration and recovery phases, and as dictated by local capacity to carry out colonoscopy. The priority on recommencement should be those qFIT-positive patients who have been 'deferred or paused', with prioritisation based on the delay they have incurred. Screening hubs will need to ensure that the backlog of cases has been sufficiently cleared, and that working capacity has been restored, before deciding to recommence qFIT invitations.

England currently offers one-off flexible sigmoidoscopy at the age of 55. Termed Bowelscope, this programme aims to detect and remove colorectal adenomas and thus prevent colorectal cancer. The future of Bowelscope screening has been under consideration for some time and neither PHE nor NHSE have been able to provide guarantees regarding the future of the programme. A national decision must be made urgently regarding the future of Bowelscope screening. It is the view of the BSG that

Bowelscope screening should not be reintroduced as this is difficult to justify during the recovery from the pandemic.

Likewise, changes to the English population-based bowel cancer screening programme, such as age extension to age 50 (the programme is currently offered to people aged 60-74) and lowering of the FIT threshold to below 120 micrograms of haemoglobin per gram of faeces (the current threshold) should take into account the degree of recovery of the service related to the current pandemic and the ability of services to expand further.

Review is required for all surveillance procedures and consideration should be given to deferring until after the deceleration and recovery phases, with subsequent gradual reintroduction in line with new BSG guidelines, as dictated by capacity. ${ }^{29}$

\section{Future work}

It is likely that the effects of the COVID-19 pandemic will continue for the foreseeable future and will significantly impact on endoscopy capacity and the ability to deliver services for a prolonged period, possibly years. There is, therefore, a need to explore safe, alternative diagnostic modalities and to reconfigure cancer diagnosis pathways. This should include consideration of novel pathways and triage tools for many patients. This will preserve endoscopic capacity for those who will benefit most, or where therapeutic interventions are likely to be required. It is, however, crucially important that long term changes to delivery of care are evidence-based.

We recommend the following as priority areas for further work:

> Coronavirus (COVID-19) screening as part of wider national work.

$>$ A national registry for contact tracing should be set up to study the risks of transmission to patients undergoing GI endoscopy and staff (see PPE and infection control measures above): this will both quality-assure the current infection control measures and inform planning for future similar emergencies.

> Less invasive endoscopy, eg wireless capsule endoscopy, should be developed by the BSG small bowel Endoscopy Quality Improvement (EQIP) team.

> Increased use of cross-sectional imaging should be developed, with the BSG working together with the British Society of Gastrointestinal and Abdominal Radiology (BSGAR) and the Association of Coloproctology GB and Ireland (ACPGBI).
> More detailed modelling of FIT levels for use in patients referred with lower GI symptoms should be developed by the BSG's lower GI EQIP team, together with NHS England and health services within the devolved administrations.

> Research should be commissioned to establish the precise AGP risks of LGI endoscopic procedures, nitrous oxide use, the administration of local anaesthetic throat spray and the insertion of enteral (NG/NJ) feeding tubes (together with the British Association for Parenteral and Enteral Nutrition [BAPEN] and BSGAR

> Endoscopy unit design, bookings processes, patient flow, and workforce and training issues will need to be addressed during the recovery phase of COVID-19 in association with Joint Advisory Group on GI Endoscopy (JAG), ACPGBI, the Association of Upper GI Surgeons (AUGIS) and other stakeholders.

\section{Conclusions}

All patients should be assessed according to the indications for endoscopy as outlined, and if they are considered to require endoscopy, they should be telephone-screened for symptoms using FTOCC/SCOTS questions. Where possible, all outpatients should undergo RT-PCR-based testing for COVID-19 virus 1-3 days prior to their procedure. Choice of PPE level should be determined by patient risk stratification, the nature of the proposed procedure and the results of patient testing. These measures should allow safe resumption of limited GI endoscopic services during the deceleration and early recovery phases of the COVID-19 pandemic.

\section{Acknowledgements}

We are grateful to Professor Greg Rubin, Dr Kevin Barrett, Professor David Sanders, Dr Brian Nicholson and Dr Stefania Chetcuti Zammit for their advice and contribution in preparing this guidance.

BSG Endoscopy Quality Improvement Programme: Colin Rees, Ian Penman, Andrew Veitch, John Anderson, Kofi Oppong, James East, Mark McAlindon.

BSG Endoscopy committee: David Nylander, Manu Nayar, Durayd Alzoubaidi, Pradeep Bhandari, Helen Griffiths, Rehan Haidry, Amyn Haji, Neil Hawkes, Bu Hayee, Srisha Hebbar, Sarah Marshall, John Morris, Ian Penman, Colin Rees, Matt Rutter, Reena Sidhu, Mo Thoufeeq, Nigel Trudgill, Eleanor Wood.

\section{References}

1 British Society of Gastroenterology. Endoscopy activity and COVID19: BSG and JAG guidance. BSG, 2020. Available from www.bsg. org.uk/covid-19-advice/endoscopy-activity-and-covid-19-bsg-andjag-guidance [Accessed 26 April 2020].

2 Chiu PWY, $\mathrm{Ng} \mathrm{SC}$, Inoue $\mathrm{H}$ et al. Practice of endoscopy during COVID-19 pandemic: position statements of the Asian Pacific Society for Digestive Endoscopy (APSDE-COVID statements). Gut 2020;69:991-6.

3 Gralnek IM, Hassan C, Beilenhoff U et al. ESGE and ESGENA position statement on gastrointestinal endoscopy and the COVID-19 pandemic. Endoscopy 2020, in press (doi: 10.1055/a-1155-6229).

4 British Society of Gastroenterology. Service recovery documents: the what, when and how. BSG, 2020. Available from www.bsg.org. uk/covid-19-advice/service-recovery-documents-the-what-whenand-how/ [Accessed 6 May 2020]. 
5 Lui RN, Wong SH, Sanchez-Luna SA et al. Overview of guidance for endoscopy during the coronavirus disease 2019 pandemic. J Gastroenterol Hepatol 2020;35:749-59.

6 Lee TJW, Siau K, Esmaily S et al. Development of a national automated endoscopy database: The United Kingdom National Endoscopy Database (NED). United Eur Gastroenterol J 2019;7:798-806

7 Lai A, Pasea L, Banerjee A et al. Estimating excess mortality in people with cancer and multimorbidity in the COVID-19 emergency. ResearchGate 2020 (doi: 10.13140/RG.2.2.34254.82242).

8 World Health Organization. WHO pandemic phase descriptions and main actions by phase. WHO, 2020. Available from www.who. int/influenza/resources/documents/pandemic_phase_descriptions_ and actions.pdf [Accessed 26 April 2020].

9 Holloway R, Rasmussen S, Zaza S et al. Updated preparedness and response framework for influenza pandemics. Centers for Disease Control and Prevention, 2014. Available from www.cdc.gov/mmwr/ preview/mmwrhtml/rr6306a1.htm.

10 British Society of Gastroenterology. GI endoscopy activity and COVID-19: Next steps. BSG, 2020. Available from www.bsg.org. uk/covid-19-advice/gi-endoscopy-activity-and-covid-19-next-steps [Accessed 6 May 2020].

11 Royal College of Physicians. Ethical dimensions of COVID-19 for frontline staff. RCP, 2020. Available from www.rcplondon.ac.uk/ news/ethical-guidance-published-frontline-staff-dealing-pandemic [Accessed 6 May 2020].

12 Zhang Y, Zhang X, Liu L, Wang H, Zhao Q. Suggestions of infection prevention and control in digestive endoscopy during current 2019-nCoV pneumonia outbreak in Wuhan, Hubei Province, China. Endoscopy 2020;52:312-4.

13 Repici A, Maselli R, Colombo M et al. Coronavirus (COVID-19) outbreak: what the department of endoscopy should know. Gastrointest Endosc 2020, in press (doi: 10.1016/j.gie.2020.03.019).

14 Soetikno R, Teoh AY, Kaltenbach T et al. Considerations in performing endoscopy during the COVID-19 pandemic. Gastrointest Endosc 2020, in press (doi: 10.1016/j.gie.2020.03.3758).

15 Repici A, Aragona G, Cengia G et al. Low risk of Covid-19 transmission in GI endoscopy. Gut 2020, in press (doi: 10.1136/gutjnl2020-321341).

16 Calderwood AH, Day LW, Muthusamy VR et al. ASGE guideline for infection control during GI endoscopy. Gastrointest Endosc 2018;87:1167-79.

17 British Society of Gastroenterology. Guidance on Decontamination of Equipment for Gastrointestinal Endoscopy: 2017 Edition. BSG, 2017. Available from www.bsg.org.uk/clinical-resource/guidanceon-decontamination-of-equipment-for-gastrointestinal-endoscopy2017-edition/
18 Han J, Wang Y, Zhu L et al. Preventing the spread of COVID-19 in digestive endoscopy during the resuming period: meticulous execution of screening procedures. Gastrointest Endosc 2020, in press (doi: 10.1016/j.gie.2020.03.3855).

19 Public Health England. COVID-19: infection prevention and control (IPC). PHE, 2020. Available from www.gov.uk/government/ publications/wuhan-novel-coronavirus-infection-prevention-andcontrol [Accessed 6 May 2020].

20 Cevik M, Bamford C, Ho A. COVID-19 pandemic - A focused review for clinicians. Clin Microbiol Infect 2020, in press (doi: 10.1016/j. cmi.2020.04.023)

21 Xiao F, Tang M, Zheng X et al. Evidence for gastrointestinal infection of SARS-CoV-2. Gastroenterology 2020;158:1831-1833.e3.

22 Lamers MM, Beumer J, van der Vaart ] et al. SARS-CoV-2 productively infects human gut enterocytes. bioRxiv 2020:2020.04.25.060350.

23 Wang X-W, Li J-WJ-SJ-F, Guo T-K et al. Excretion and detection of SARS coronavirus and its nucleic acid from digestive system. World J Gastroenterol 2005;11:4390-5.

24 Wang X-W, Li J-WJ-SJ-F, Guo T-K et al. Concentration and detection of SARS coronavirus in sewage from Xiao Tang Shan Hospital and the 309th Hospital. J Virol Methods 2005;128:156-61.

25 Chan KH, Poon LLLM, Cheng VCC et al. Detection of SARS coronavirus in patients with suspected SARS. Emerg Infect Dis 2004;10:294-9.

26 Zang R, Castro MFG, McCune BT et al. TMPRSS2 and TMPRSS4 mediate SARS-CoV- 2 infection of human small intestinal enterocytes. bioRxiv 2020:2020.04.21.054015.

27 Rhatigan E, Tyrmpas I, Murray G, Plevris JN. Scoring system to identify patients at high risk of oesophageal cancer. $\mathrm{Br}$ J Surg 2010;97:1831-7.

28 British Society of Gastrointestinal and Abdominal Radiology. CT Colonography activity and Covid-19: British Society of Gastrointestinal and Abdominal Radiology guidance. BSGAR, 2020. Available from www.bsgar.org/society/covid-19-and-bsgarupdates-1/ [Accessed 7 May 2020].

29 Rutter MD, East J, Rees C] et al. British Society of Gastroenterology/ Association of Coloproctology of Great Britain and Ireland/Public Health England post-polypectomy and post-colorectal cancer resection surveillance guidelines. Gut 2020;69:201-23.

Address for correspondence: Prof Colin J Rees, Population Health Sciences Institute, Newcastle University Centre for Cancer, Newcastle University, Newcastle Upon Tyne NE2 4HH, UK.

Email:colin.rees@newcastle.ac.uk 